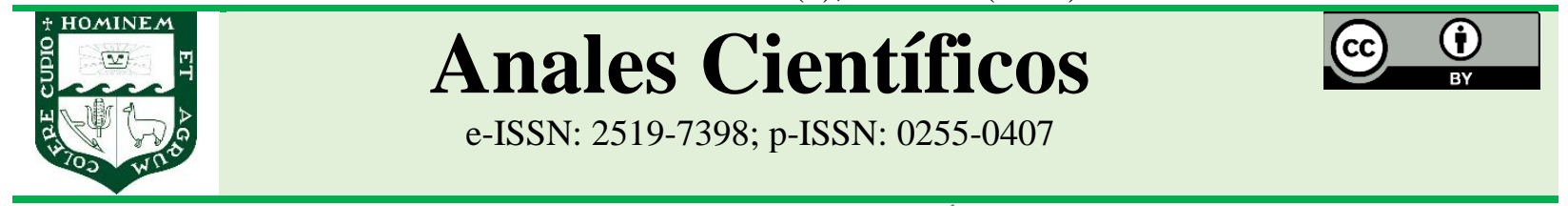

ARTÍCULO ORIGINAL - RESEARCH ARTICLE http://dx.doi.org/10.21704/ac.v82i2.1791

\title{
EFECTO DEL CAFÉ FERMENTADO CON BIOPRODUCTO SOBRE LA GERMINACIÓN DE SEMILLAS Y EL DESARROLLO DE POSTURAS
}

\section{Effect of coffee fermented with bioproduct on seed germination and seedling development}

\author{
Yusdel Ferrás Negrín ${ }^{1 *}$ (D); Carlos Bustamante González ${ }^{2}$ (D); Maykelis Díaz Solares ${ }^{3}$ (D); \\ Ciro Sánchez Esmori ${ }^{1}$ iD \\ ${ }^{1}$ Instituto de Investigaciones Agro-Forestales (INAF), Unidad de Ciencia y técnica de Base (UCTB) Jibacoa, Manicaragua, \\ Villa Clara, Cuba. \\ ${ }^{2}$ Instituto de Investigaciones Agro-Forestales (INAF), Unidad de Ciencia y técnica de Base (UCTB) Cruce de los Baños, \\ Tercer Frente, Santiago de Cuba, Cuba. \\ ${ }^{3}$ Estación Experimental de Pastos y Forrajes "Indio Hatuey" Central España Republicana, Matanzas, Cuba. \\ *E-mail: yusdel@jibacoa.inaf.co.cu
}

\begin{abstract}
IHPLUS is a bioproduct based on efficient microorganisms that constitute an option for the development of cultures. The objective of the research was to evaluate the effect of coffee fermented with IHPLUS on seed germination and posture development. After the pulping of the coffee, the fermentation was carried out in four concentrations with IHPLUS (5\%, 10\%, 15\% and 20\%), and a control with water alone. It was evaluated the percentage of germination of the seeds; the height, the length of the root and the dry mass of the seedlings when they had cotyledonous leaves; and the height, the diameter of the stem, the foliar area and the dry mass of the seedlings at the end of their revival. By fermenting the seeds in a medium with a higher concentration of bioproduct, germination was increased, the $15 \%$ and $20 \%$ treatments increased this indicator by 13,4 and $16,1 \%$ respectively compared to the control. In germinators, the seedlings had better morpho-physiological characteristics with the concentrations of the bioproduct, especially with $15 \%$. In the positions, the $15 \%$ concentration increased by 17,$9 ; 26,8 ; 50$ and $38,6 \%$ height, stem diameter, dry mass and leaf area respectively compared to the control. The IHPLUS in the fermentation of coffee seeds significantly improved the percentage of germination, the quality indicators of the seedlings in the germinators and of the positions at the end of their revival. The $15 \%$ concentration was the most comprehensive and recommended variant to use.
\end{abstract}

Keywords. Coffea canephora | IHPLUS | efficient microorganisms | seedlings | seed

\section{RESUMEN}

El IHPLUS es un bioproducto a base de microorganismos eficientes que constituyen una opción para el desarrollo de los cultivos. La investigación tuvo como objetivo evaluar el efecto del café fermentado con IHPLUS sobre la germinación de semillas y el desarrollo de posturas. Posterior al despulpe del café la fermentación se realizó en cuatro concentraciones con IHPLUS $(5 \%, 10 \%, 15 \%$ y $20 \%)$, y un testigo con agua sola. Se evaluó el por ciento de 
germinación, la altura, el largo de la raíz y la masa seca de las plántulas cuando tuvieron las hojas cotiledonares, y la altura, el diámetro del tallo, el área foliar y la masa seca de las posturas al finalizar su aviveramiento. Al fermentar las semillas en un medio con mayor concentración de bioproducto se aumentó la germinación, los tratamientos 15\% y $20 \%$ incrementaron este indicador en un 13,4 y $16,1 \%$ respectivamente en comparación al testigo. En germinadores las plántulas tuvieron mejores características morfo-fisiológicas con las concentraciones del bioproducto, sobre todo con el 15\%. En las posturas la concentración del 15\% incrementó en un 17,9; 26,8; 50 y $38,6 \%$ la altura, el diámetro del tallo, la masa seca y el área foliar respectivamente en comparación al testigo. El IHPLUS en la fermentación de las semillas de café mejoró significativamente el por ciento de germinación, los indicadores de calidad de las plántulas en los germinadores y de las posturas al concluir su aviveramiento. La concentración del $15 \%$ fue la variante más integral y recomendable a utilizar.

Palabras clave: Coffea canephora | IHPLUS | microorganismos eficientes | plántulas | semilla

Forma de citar el artículo (Formato APA):

Ferrás, Y., Bustamante, C., Díaz, M., \& Sánchez, C. (2021). Efecto del café fermentado con bioproducto sobre la germinación de semillas y el desarrollo de posturas. Anales Científicos. 81(2), 288-295. http://dx.doi.org/10.21704/ac.v82i2.1791

Autor de correspondencia (*): Yusdel Ferrás Negrín. Email: yusdel@jibacoa.inaf.co.cu

(C) Los autores. Publicado por la Universidad Nacional Agraria La Molina.

This is an open access article under the CC BY

\section{INTRODUCCIÓN}

Las especies de café cultivadas comercialmente son Coffea arabica L. y Coffea canephora Pierre ex Froehner, las que aportan el 70 y $30 \%$ respectivamente de la cosecha mundial (Ortiz et al., 2018).

La buena calidad de la semilla utilizada en las siembras de café es el primer paso para garantizar el desarrollo óptimo del cultivo (Velásquez et al., 2003). Se considera como uno de los principales factores que influyen en la producción de plantas vigorosas en condiciones de campo, lo que equivale a una mayor productividad del cultivo (González et al., 2015).

Entre los factores que afectan la calidad de las semillas se encuentran la variedad, las condiciones de cultivo, la cosecha en el punto apropiado de madurez fisiológica, el procesamiento adecuado que no cause daño mecánico a las semillas por fracturas o fricciones, las condiciones de secado, el contenido de humedad final y la edad de las semillas (Velásquez et al., 2003). La germinación y la fermentación de los granos del café son dos bioprocesos que tienen lugar durante el tratamiento posterior a la cosecha. El proceso de germinación se inicia mientras estas semillas no ortodoxas todavía están dentro de la cereza (Waters et al., 2017).
Vera (1994) expresó que en la variedad de café Caturra Rojo, los tiempos de fermentación inicialmente no influyeron sobre el comportamiento fisiológico de las semillas; sin embargo, cuando estas fueron almacenadas por tiempos prolongados (ocho meses) observó una marcada diferencia obteniéndose como punto máximo de fermentación entre 20 y 21 horas. Además, se refirió a que la sobre fermentación del grano puede afectar el embrión y la buena presencia de las semillas.

Los inoculantes microbianos mejoran la productividad del sistema agropecuario a largo plazo y se considera una tecnología limpia, alineada con principios de la agricultura sustentable, frente al aumento abusivo de la utilización de pesticidas y fertilizantes en estos últimos tiempos. Varios microorganismos son utilizados en la práctica agrícola habitual, y otros tienen potencial para ser utilizados en el futuro (Naiman et al., 2009; Soroa et al., 2009; citados por Castillo et al., 2014).

León et al. (2016) expresaron que en la actualidad la producción orgánica está usando de forma acelerada los microorganismos eficientes (ME), en la agricultura. Estos microorganismos han sido ampliamente usados con diferentes propósitos, demostrando que mejoran la calidad de los suelos; cuando entran en contacto con la materia orgánica segregan sustancias beneficiosas tales como: vitaminas, ácidos orgánicos, minerales, y son los principales responsables de la descomposición 
de la misma. Además, se estima que son una fuente prometedora de sustancias bioactivas, debido a su capacidad de producir metabolitos secundarios e incremento de la biodiversidad microbiana.

Se ha constatado que los ME son estudiados en numerosos países, entre ellos Cuba, donde se han realizado trabajos para su aplicación y generalización en diversos cultivos y otras actividades como la producción animal, actividades domésticas y de servicio a instalaciones turísticas entre otras.

El IHPLUS ${ }^{\circledast}$, es un bioproducto de amplio uso agropecuario basado en microorganismos nativos. Se deriva de la introducción, adaptación y diseminación de la tecnología desarrollada en Japón, que utiliza una mezcla de microorganismos eficientes como biofertilizante, probiótico, antiséptico, y limpieza de residuales líquidos de la agricultura y el turismo. Adaptada al utilizar microorganismos de estratos bajos de bosques no perturbados o poco intervenidos por el hombre, en Cuba y sustratos nacionales locales. Lograda en la Estación Experimental Indio Hatuey con marca registrada por la Oficina Nacional de la Propiedad Industrial (Díaz et al., 2020).

En la actualidad no se cuenta con información sobre el efecto que puede ejercer la adición del IHPLUS en la germinación de semillas de café, cuando se aplica en el proceso de fermentación. Teniendo en cuenta que Waters et al. (2017) expresaron que el proceso de germinación es asíncrono y la evolución de la germinación depende de cómo se procesan las mismas se realizó este trabajo con el objetivo de evaluar el efecto del café fermentado con IHPLUS sobre la germinación de semillas y el desarrollo de posturas.

\section{MATERIALES Y MÉTODOS}

El estudio se realizó durante la campaña de vivero 2017-2018 en la Estación Experimental Agro-Forestal de Jibacoa ubicada en el municipio de Manicaragua, Villa Clara, Cuba.

Se utilizaron semillas de la especie Coffea canephora Pierre ex Froehner, variedad 'Robusta'.
Las cerezas fueron despulpadas en una despulpadora Penagos $^{\circledR}$ modelo DH-4. La fermentación fue mediante un proceso húmedo por un tiempo de 24 horas. Se usó igual volumen de semillas y agua o solución agua-ME según los tratamientos:

1) Semillas fermentadas en agua sola, $0 \%$ de concentración con IHPLUS (Testigo);

2) semillas fermentadas en agua con IHPLUS al 5\%;

3) semillas fermentadas en agua con IHPLUS al 10\%;

4) semillas fermentadas en agua con IHPLUS al $15 \%$;

5) semillas fermentadas en agua con IHPLUS al $20 \%$.

El bioproducto IHPLUS procedió de la Estación Experimental de Pastos y Forrajes de Indio Hatuey. Este centro de investigación desarrolla la producción del mismo a base de ME, según los principios propuestos por Higa y Parr (1994) (Álvarez y Damião, 2018).

Las semillas después de fermentadas según los tratamientos descritos fueron lavadas y oreadas durante tres días a la sombra en un lugar fresco.

En germinadores con arena de río se sembraron tres repeticiones de cada tratamiento con 100 semillas distribuidas en bloque completamente aleatorizado.

Cuando las plántulas tuvieron hojas cotiledonares se trasplantaron 20 de cada tratamiento a bolsas de $14 \mathrm{~cm}$ $\mathrm{x} 22 \mathrm{~cm}$ distribuidos en un diseño completamente aleatorizado.

El sustrato estuvo compuesto por tres partes de suelo Fersialítico Pardo Rojizo y una parte de abono orgánico. Se consideró adecuado para la producción de posturas de cafeto y se caracterizó por ser ligeramente ácido $(\mathrm{pH} \mathrm{KCl} \mathrm{5,9),} \mathrm{tener} \mathrm{altos} \mathrm{contenidos} \mathrm{de} \mathrm{materia}$ orgánica $(5,83 \%)$, de potasio $(93,8 \mathrm{mg} / 100 \mathrm{~g})$ y medios de fósforo $(15,9 \mathrm{mg} / 100 \mathrm{~g})$.

Evaluaciones en germinadores

a) Germinación de las semillas (\%): se determinó por la metodología propuesta por Ranal et al. (2009) con el uso de la hoja de cálculo Excel.

Cuando el $90 \%$ de las plántulas tuvieron hojas cotiledonares, a 20 de ellas por cada tratamiento se evaluó: 
b) Altura del hipocótilo: se midió con una regla graduada desde la base del hipocótilo de la plántula hasta las hojas cotiledonares $(\mathrm{cm})$.

c) Largo de la raíz: se midió con una regla graduada desde la base del hipocótilo de la plántula hasta la terminación de la raíz $(\mathrm{cm})$.

d) Masa seca: las plántulas se colocaron en una estufa a $65^{\circ} \mathrm{C}$ hasta alcanzar masa seca constante $(\mathrm{g})$.

Evaluaciones en vivero

Cuando las posturas tuvieron 6 pares de hojas, a 10 de ellas por cada tratamiento se evaluó:

e) Altura: se midió con una regla graduada desde la base del tallo de la planta hasta el ápice $(\mathrm{cm})$.

f) Diámetro del tallo: se midió con un pie de rey a $1 \mathrm{~cm}$ de la base del tallo (cm).

g) Masa seca: las plantas se colocaron en una estufa a $65^{\circ} \mathrm{C}$ hasta alcanzar masa seca constante $(\mathrm{g}) . \mathrm{h}$ ) Área foliar (AF): se determinó con el uso de la fórmula de González et al. (2000) citados por Pérez et al. (2002) (AF= largo x ancho x 0,67), se expresó en $\mathrm{cm}^{2}$.

A los datos se les realizó la prueba de normalidad y homogeneidad de varianza. La comparación de las medias se realizó mediante la prueba de rangos múltiples de Duncan con un nivel de confianza del
95\%. Los valores porcentuales fueron transformados previamente al análisis a arcosen $\sqrt{ } \mathrm{p}$, y se muestran en la tabla de los resultados los valores sin transformar. El procesamiento estadístico de los datos se realizó con el programa InfoStat versión 1.0.

Se realizó análisis de regresión entre las concentraciones de IHPLUS utilizadas en la fermentación de las semillas de cafeto y las variables dependientes evaluadas cuando existió correlaciones significativas entre ellas. Se usó el programa STATISTICA versión 8.0.

\section{RESULTADOS Y DISCUSIÓN}

Existió un efecto positivo y significativo de las concentraciones de IHPLUS utilizadas en la fermentación de las semillas de café sobre el por ciento de germinación. Este bioproducto al $15 \%$ y $20 \%$ fueron las únicas variantes que incrementaron significativamente el por ciento de germinación en un $13,4 \%$ y $16,1 \%$ respectivamente en comparación al testigo, sin diferencias del resto de las concentraciones (Figura 1).

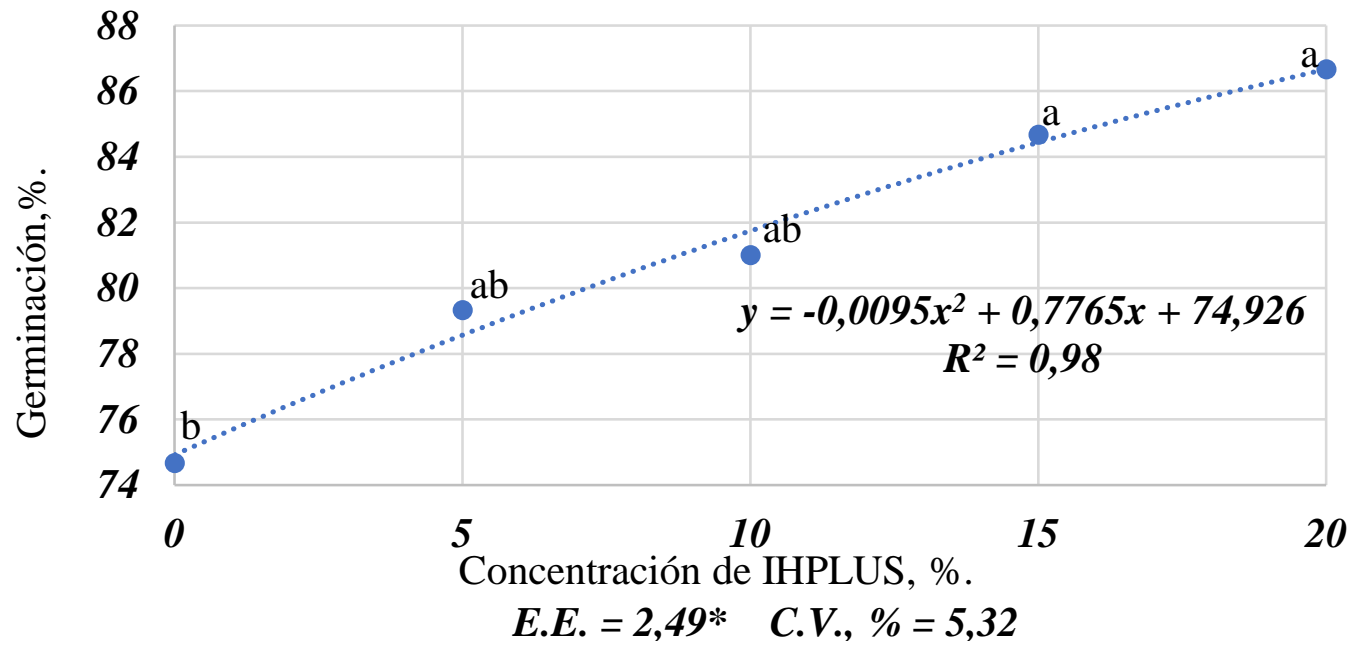

Figura 1. Influencia de las concentraciones del IHPLUS en la germinación de las semillas. *Puntos con letras diferentes sus medias difieren significativamente para valor de $p \leq 0,05$ según prueba de Duncan.

El por ciento de germinación fue la única variable que presentó correlación significativa con las concentraciones de IHPLUS utilizadas en la fermentación. Con el aumento del bioproducto se incrementó significativamente esta variable con un $\mathrm{R}^{2}$ del 0,98 (Figura 1). 
Durante la fermentación se producen varios cambios fisiológicos en los granos de café. Los microorganismos (levadura, bacterias y hongos) juegan un papel importante en la degradación del mucílago al producir varias enzimas, alcoholes y ácidos durante el proceso de fermentación (Haile y Hee, 2019). En este sentido la adición del IHPLUS a la fermentación de las semillas pudo favorecer este proceso, facilitar la obtención de semillas más limpias de mucílago y contribuir a su mejor germinación. Bytof et al. (2007) expresaron que en el proceso húmedo se elimina la pulpa, lo que permite el inicio de la germinación de la semilla. La confirmación de que las semillas de café germinan durante su procesamiento se estableció recientemente sobre la base de la expresión del isocitrato liasa específica de germinación y un aumento en la abundancia de btubulina, un marcador para la división o elongación celular.

Estos resultados también pudieron estar motivados por el efecto de las sustancias estimuladoras presente en el bioproducto como resultado del propio metabolismo de los microorganismos, además a la posible degradación del pergamino facilitando su germinación. En este sentido, Loarte (2017) y Tanya y Leiva (2019) se refirieron a que los ME incrementan el porcentaje de germinación en semilleros, por su efecto hormonal similar al del ácido giberélico; además Rostrán y Bárcenas, (2016) expresaron que las bacterias ácido lácticas aumentan la fragmentación de los componentes de la materia orgánica, tales como la lignina y la celulosa, compuestos estos presentes en el pergamino del café. Luna y Mesa (2016) plantearon que los microorganismos eficientes promueven y aseguran una mejor germinación y desarrollo de las plantas.

Los porcentajes de germinación de esta investigación se relacionan a los obtenidos por Ortíz et al. (2018) en Coffea canephora, quienes alcanzaron a los 50 días un $75 \%$ de germinación en las semillas sumergidas en agua durante 24 horas, que incrementó hasta el $87 \%$ al sumergirlas durante una hora a $40^{\circ} \mathrm{C}$.

Cuando se fermentaron las semillas de café solamente en agua (testigo), se tuvieron los valores medios absolutos más bajos en las características de las plántulas en los germinadores (Tabla 1).
En el largo de la raíz y la altura de las plántulas se pudo constatar que entre las concentraciones no existieron diferencias significativas. En la primera variable las concentraciones $5 \%$ y $15 \%$ se diferenciaron del testigo con incremento de $12 \%$ y $17,3 \%$ respectivamente; y en la segunda el testigo sí fue inferior significativamente a los demás tratamientos.

En la masa seca de las plántulas, entre las concentraciones $5 \% ; 10 \%$ y $15 \%$ no existieron diferencias significativas, con estas variantes se obtuvieron incrementos de un $25,4 \% ; 27 \%$ y $34,9 \%$ respectivamente en comparación al testigo.

En sentido general la concentración del $15 \%$ de IHPLUS presentó los valores medios más elevados, lo cual justifica su uso si tenemos presente los criterios de Velásquez et al. (2003) cuando expusieron que una semilla de café de buena calidad entre otras características debe tener buen vigor de las plántulas al germinar y un sistema radical bien conformado.

Los ME incrementan el vigor y crecimiento del tallo y raíces, desde la germinación hasta la emergencia de las plántulas, por su efecto como rizo bacterias promotoras del crecimiento vegetal y aumenta las probabilidades de supervivencia de las plántulas (Loarte, 2017; Tanya y Leiva, 2019).

Las características morfológicas de las posturas de cafeto mostraron una respuesta significativa a las concentraciones de IHPLUS donde fueron fermentadas las semillas de café (Tabla 2).

La concentración del 15\% propició el mejor desarrollo integral de las posturas de café e incrementó en 17,9; 26,$8 ; 50$ y $38,6 \%$ la altura, el diámetro del tallo, la masa seca y el área foliar respectivamente con respecto al testigo. Esta concentración en la altura no se diferenció del $5 \%$ y $10 \%$, de igual forma sucedió en el diámetro del tallo con la del 10\%; sin embargo, en la masa seca y el área foliar ningún tratamiento se le asemejó.

Cupull et al. (2003) obtuvieron incrementos significativos en la altura, diámetro del tallo, número de pares de hojas y masa seca foliar en posturas de Coffea arabica L. var. 'Caturra Rojo' al utilizar Trichoderma viride en el momento de la siembra. 
Utria et al. (2004), obtuvieron el mejor desarrollo en posturas Coffea arabica L. var 'Caturra Rojo' al embeber las semillas con brasinoesteroide con respecto al tratamiento control, pero con tendencia a tener mejores respuestas cuando los mismos fueron asperjados en el segundo par de hojas verdaderas en concentraciones de 0,01 y $0,05 \mathrm{mg} \mathrm{L}^{-1}$. Estos autores expusieron que los bioestimulantes pueden ejercer su actividad en diferentes órganos, momentos y estadios de los cultivos.

Luna y Mesa (2016) expresaron que los ME aumentan la capacidad fotosintética de los cultivos, e informaron los resultados positivos obtenidos en varias investigaciones con la implementación de esta tecnología, en este sentido se refirieron a la influencia de los ME sobre: la precocidad de la cosecha y el número de flores por planta en el pepino (Cucumis sativus, L. híbrido Atar Ha-435); incrementos de 1,5 t $\mathrm{ha}^{-1}$ en los rendimientos agrícolas del frijol con la aplicación de $10 \mathrm{~L} \mathrm{ha}^{-1}$ de ME con respecto a las plantas no biofertilizadas; en lechuga (Lactuca sativa, L.), al aplicar una dosis de $48 \mathrm{~L} \mathrm{ha}^{-1}$ del producto, se obtuvieron aumentos en la altura y el peso de la roseta de hojas y una reducción del ciclo del cultivo; y en la fruta bomba (Carica papaya, L.), se aumentó la altura y grosor del tallo, lo que motivó a un adelanto con relación al testigo, entre siete y 10 días.

Tabla 1. Influencia del IHPLUS en las características de las plántulas de café con hojas cotiledonares.

\begin{tabular}{llll}
\hline $\begin{array}{c}\text { Concentraciones de } \\
\text { IHPLUS, \% }\end{array}$ & \multicolumn{1}{c}{ Largo de la raíz, cm } & \multicolumn{1}{c}{ Altura, cm } & \multicolumn{1}{c}{ Masa seca, g } \\
\hline Testigo (0) & $6,64 \mathrm{~b}$ & $5,54 \mathrm{~b}$ & $0,063 \mathrm{c}$ \\
$\mathbf{5}$ & $7,44 \mathrm{a}$ & $5,94 \mathrm{a}$ & $0,079 \mathrm{ab}$ \\
$\mathbf{1 0}$ & $7,26 \mathrm{ab}$ & $6,06 \mathrm{a}$ & $0,080 \mathrm{ab}$ \\
$\mathbf{1 5}$ & $7,79 \mathrm{a}$ & $6,19 \mathrm{a}$ & $0,085 \mathrm{a}$ \\
$\mathbf{2 0}$ & $7,34 \mathrm{ab}$ & $5,97 \mathrm{a}$ & $0,074 \mathrm{~b}$ \\
E.E. & $0,26^{*}$ & $0,13^{*}$ & $0,003^{* *}$ \\
C.V., \% & 15,04 & 9,59 & 14,31
\end{tabular}

*; **medias con letras diferentes en una misma columna difieren significativamente para valor de $p \leq 0,05$ y 0,01 respectivamente.

Tabla 2. Influencia del IHPLUS en las características morfo-fisiológicas de las posturas de café.

\begin{tabular}{|c|c|c|c|c|}
\hline $\begin{array}{l}\text { Concentraciones } \\
\text { IHPLUS, \% }\end{array}$ & de Altura, cm. & $\begin{array}{l}\text { Diámetro del tallo, } \\
\text { cm }\end{array}$ & Masa seca total, $\mathrm{g}$ & Área Foliar, $\mathbf{c m}^{2}$ \\
\hline Testigo (0) & $29,21 \mathrm{~b}$ & $0,41 \mathrm{~b}$ & $3,14 \mathrm{c}$ & $421,12 b$ \\
\hline 5 & $30,47 \mathrm{ab}$ & $0,42 \mathrm{~b}$ & $2,80 \mathrm{c}$ & $428,09 \mathrm{~b}$ \\
\hline 10 & $31,99 \mathrm{ab}$ & $0,46 \mathrm{ab}$ & $3,90 \mathrm{~b}$ & $489,44 \mathrm{~b}$ \\
\hline 15 & $34,43 \mathrm{a}$ & $0,52 \mathrm{a}$ & $4,71 \mathrm{a}$ & 583,81 a \\
\hline 20 & $28,46 \mathrm{~b}$ & $0,43 \mathrm{~b}$ & $3,01 \mathrm{c}$ & $428,68 \mathrm{~b}$ \\
\hline E.E. & $1,19 * *$ & $0,02 * *$ & $0,26^{*}$ & $23,05 * *$ \\
\hline C.V., \% & 12,21 & 15,32 & 23,51 & 15,50 \\
\hline
\end{tabular}

Estos resultados indican que el procedimiento postcosecha para la obtención de semillas de C. canephora variedad 'Robusta' juega un papel importante. El tratamiento que se les haga desde su fermentación para retirar el mucílago puede tener influencia positiva en la germinación, en las características de las plántulas en los germinadores y por ende en el desarrollo de las posturas de café durante su aviveramiento. Lo antes expuesto corrobora lo expresado por Selmar et al.
(2006) cuando evidenciaron que la germinación se inicia en las semillas de café durante el curso de los tratamientos estándar de post-cosecha. El alcance y la naturaleza de los procesos de germinación dependen del método de procesamiento. 


\section{CONCLUSIONES}

El bioproducto IHPLUS en la fermentación de las semillas de café incrementó el por ciento de germinación, los indicadores de calidad de las plántulas en los germinadores y de las posturas al concluir la etapa de aviveramiento. La concentración del $15 \%$ fue la variante más integral y recomendable a utilizar.

\section{Conflictos de intereses}

Los autores del presente trabajo de investigación no tienen ningún potencial conflicto de interés personal o económico con otras personas u organizaciones que puedan influir indebidamente con el presente manuscrito.

\section{Contribuciones de los autores}

Preparación y ejecución: YFN, CBG; Desarrollo de la metodología: YFN, CBG; Concepción y diseño: YFN, MDS; Edición del artículo: YFN, CBG, CSE; Supervisión del estudio: MDS

\section{REFERENCIAS}

- Álvarez, J. L. \& Damião, J.C. (2018). Producción de posturas de café con la aplicación de microorganismos eficientes en Angola. Centro Agrícola, 45(2), 29-33. http://scielo.sld.cu/pdf/cag/v45n2/cag04218.pdf

- Bytof, G., Knopp, S.E., Kramer, Daniela, Breitenstein, B., Bergervoet, J. H.W., Groot, S.C. $\&$ Selmar, D. (2007). Transient Occurrence of Seed Germination Processes during Coffee Post Harvest Treatment. Annals of Botany, 100, 61-66. https://www.researchgate.net/publication/6350862 _Transient_Occurrence_of_Seed_Germination_Pr ocesses_during_Coffee_Post_Harvest_Treatment

- Castillo, F., Sánchez, J.D., Rangel, S.E. \& Canul, J. (2014). Efecto de microorganismos en la promoción de la germinación de semillas de la cactácea Echinocactus platyacanthus LINK \& OTTO. Interciencia, 39(12), 863-867. http://www.redalyc.org/pdf/339/33932786006.pdf

- Cupull, R., Andreu, C.M., Pérez, C., Delgado, Y. \& Cupull, M.C. (2003). Efecto de Trichoderma viride como estimulante de la germinación, en el desarrollo de posturas de cafetos y el control de
Rhizoctonia solani Kuhn. Centro Agricola, 30(1), 21-25.

http://cagricola.uclv.edu.cu/descargas/pdf/V30Numero_1/cag061031266.pdf

- Díaz, M., Martín, G.J., Miranda, T., Fonte, L., Lamela, L., Montejo, I. L., Contino, Y., Ojeda, F., Medina, R., Ramírez, W. M., Lezcano, L. C., Pentón, G., Peter, H., Alonso, O., Catalá, R., \& Milera M. C. (2020, 13 de marzo). Obtención y utilización de microorganismos nativos: el bioproducto IHPLUS. [Sesión de conferencia]. Proyecto Biocarbono Cuba. https://www.researchgate.net/publication/3399162 60_Obtencion_y_utilizacion_de_microorganismos _nativos_el_bioproducto_IHPLUS_R

- González, M. E., Rosales, P. J., Castilla, Y., Lacerra, J. A.; \& Ferrer, M. (2015). Effect of bioenraiz $^{\circledR}$ as stimulant of coffee plants (coffea arabica L.) germination and the development. Cultivos Tropicales, 36(1), 71-77. http://scielo.sld.cu/pdf/ctr/v36n1/en_ctr09115.p df

- Haile, M. \& Hee, W. (2019). The Role of Microbes in Coffee Fermentation and Their Impact on Coffee Quality. Journal of Food Quality. 2019, Article ID 4836709,

6. https://www.hindawi.com/journals/jfq/2019/4836 709/

- León, R., Torres, A., Peñarrieta, S., Mero, J. \& Fosado, O. (2016). Efecto de la materia orgánica y microorganismos eficientes en el comportamiento productivo de la Acelga. Revista Espamciencia, $7(2)$, 127-134. http://investigacion.espam.edu.ec/index.php/Revis ta/article/viewFile/236/175

- Loarte, L.A. (2017). Evaluación de tres tipos de bocashi con la aplicación de microorganismos eficientes, elaborados con residuos orgánicos de las UPAs de la parroquia Chuquiribamba, del Cantón Loja. [Tesis de candidatura. Facultad Agropecuaria y de Recursos Naturales Renovables carrera de Ingeniería Agronómica, Universidad Nacional de Loja-Ecuador].

http://dspace.unl.edu.ec/jspui/bitstream/12345678 9/19242/1/Lelis\%20Aleida\%20Loarte\%20Enr\%C 3\%ADquez.pdf.

- Luna, M. A., \& Mesa, J. R. (2016). Microorganismos eficientes y sus beneficios para 
los agricultores. Agroecosistemas, 4(2), 31-40. https://aes.ucf.edu.cu/index.php/aes/article/view/8 $4 / 115$

- Ortiz, V., Ordaz, V.M., Aldrete, A., Escamilla, E., Sánchez, G. \& López, R.M. (2018). Tratamientos pregerminativos en semillas de dos especies del género Coffea. Agroproductividad, 11(4), 68-73. http://revista-

agroproductividad.org/index.php/agroproductivida d/article/view/272/201

- Pérez, A., Bustamante, C., Rodríguez, R., Díaz, A., Bertot, Y. \& Rodríguez, M.I. (2002). Influencia de diferentes variantes de fertilización en el crecimiento y desarrollo de posturas de Coffea canephora Pierre. Cultivos Tropicales, 23(4), 8993.

https://www.redalyc.org/pdf/1932/193218135012. pdf

- Ranal, M.A., Garcia, D., Resende, W., \& Mendes, C. (2009). Calculating germination measurements and organizing spreadsheets, Revista Brasil. Bot., $32(4)$, 849-855. https://www.scielo.br/j/rbb/a/Ybz4PfzG4kVdQZH nKmxvbdr/?lang=en\&format=pdf

- Rostrán, J.L. \& Bárcenas, M.J. (2016). Manual de producción mica (EM, microorganismos de montaña). "Abonos orgánicos la base para los sistemas de producción sustentables". En J.L. Rostrán, M.J. Bárcenas, X. Castillo, J.E. Escobar, K. Naruo \& T. Tajiri. Manual de Abonos Orgánicos (pp. 38-45). Universidad Nacional Autónoma de Nicaragua (UNAN - LEÓN). http://riul.unanleon.edu.ni:8080/jspui/bitstream/12 3456789/5517/1/CNRA0002.pdf

- Selmar, D., Bytof, G., Knopp, S. E. \& Breitenstein, B. (2006). Germination of coffee seeds and its significance for coffee quality. Plant Biology, 8(2), 260-264.

https://eurekamag.com/research/004/435/0044353 39.php

- Tanya, M \& Leiva, M. (2019). Microorganismos eficientes, propiedades funcionales y aplicaciones agrícolas. Centro Agrícola, 46(2), 93-103. http://scielo.sld.cu/pdf/cag/v46n2/0253-5785-cag46-02-93.pdf

- Utria, E., Rodríguez, V., Moisés, L. G., Calderón, J. O., Suárez, F. (2004). Respuesta de plántulas de cafeto (Coffea arabica L.) a la aplicación de brasinoesteroide en diferentes concentraciones y etapas de su desarrollo. Revista Chapingo Serie Horticultura, $\quad 10(1), \quad 11-14$. https://chapingo.mx/revistas/revistas/articulos/doc /rchshX170.pdf

- Velásquez, G.P., Arcila, J., Aristizábal, M. (2003). Relación entre el proceso de beneficio de la semilla de café (Coffea arabica var. Colombia) y el disturbio de la raíz bifurcada. Cenicafé, 54(4), 316$328 . \quad \mathrm{http} / / /$ infocafes.com/portal/wpcontent/uploads/2015/12/relacion-entre-elproceso-de-beneficio-de-la-semilla-de-cafe.pdf

- Vera, J.L. (1994). Estudio de factores que afectan la viabilidad de las semillas de café (Coffea arabica L. Var. Caturra Rojo). [Tesis de candidatura para optar al título de ingeniero agrónomo, Universidad técnica de Manabi, Ecuador]. https://books.google.com.cu/books?id=EZQzAQA AMAAJ\&pg=PP9\&lpg=PP9\&dq=influencia + de + fermentaci\%C3\%B3n+en+la+germinaci\%C3\%B3 $\mathrm{n}+\mathrm{del}+\mathrm{caf} \% \mathrm{C} 3 \%$ A9\&source $=\mathrm{bl} \&$ ots $=61 \mathrm{vX} 4 \mathrm{n} \_\mathrm{cX}$ i\&sig=ACfU3U0OEZVDfeHC4ThugBnEkJTNb $\mathrm{NcOCA} \& \mathrm{hl}=\mathrm{es} \& \mathrm{sa}=\mathrm{X} \& \mathrm{ved}=2 \mathrm{ahUKEwiFxMrxr}$ foAhXidd8KHTb2BBAQ6AEwAHoECAwQKQ\# $\mathrm{v}=$ onepage $\& \mathrm{q}=$ influencia $\% 20 \mathrm{de} \% 20$ fermentaci $\%$ C3\%B3n\%20en\%20la\%20germinaci\%C3\%B3n\% 20del $\% 20$ caf $\%$ C $3 \%$ A9\&f $=$ false

- Waters, D.M., Arendt, E. K. \& Moroni, A. V. (2017). Overview on the mechanisms of coffee germination and fermentation and their significance for coffee and coffee beverage quality. Food Science and Nutrition, 57(2), 259-274. https://www.tandfonline.com/doi/citedby/10.1080/ 10408398.2014.902804?scroll=top\&needAccess= true. 